“C 2004 IEEE. Personal use of this material is permitted. Permission from IEEE must be obtained for all other uses, in any current or future media, including reprinting/republishing this material for advertising or promotional purposes, creating new collective works, for resale or redistribution to servers or lists, or reuse of any copyrighted component of this work in other works." 


\section{An Efficient Algorithm for Line Extraction from Laser Scans}

\author{
Alen Alempijevic \\ ARC Centre of Excellence in Autonomous Systems \\ (CAS) \\ University of Technology Sydney \\ NSW, Australia \\ alalemp@eng.uts.edu.au
}

\author{
Gamini Dissanayake \\ ARC Centre of Excellence in Autonomous Systems \\ (CAS) \\ University of Technology Sydney \\ NSW, Australia \\ gdissa@eng.uts.edu.au
}

\begin{abstract}
In this paper, an algorithm for extracting line segments from information gathered by a laser rangefinder is presented. The range scan is processed to compute a parameter that is invariant to the position and orientation of straight lines present. This parameter is then used to identify observations that potentially belong to straight lines and compute the slope of these lines. Log-Hough transform, that only explores a small region of the Hough space identified by the slopes computed, is then used to find the equations of the lines present. The proposed method thus combines robustness of the Hough transform technique with the inherent efficiency of line fitting strategies while carrying out all computation in the sensor coordinate frame yielding a fast and robust algorithm for line extraction from laser range scans. Two practical examples are presented to demonstrate the efficacy of the algorithm and compare its performance to the traditional techniques.
\end{abstract}

Keywords- laser rangefinders; line segment extraction; Hough transform; robot navigation

\section{INTRODUCTION}

Laser rangefinders have become extremely popular sensors for robot navigation, particularly in indoor environments. Extracting geometric features present in the environment by processing the raw sensor data is often an integral part of many navigation algorithms. Straight lines are usually the most predominant geometric feature in typical indoor environments. Matching natural features such as straight lines or corners extracted from sensor data with information available in a map of the environment form the basis of Kalman filter based localization algorithms. Furthermore, in situations where a map of the environment is not available, feature based simultaneous localization and mapping algorithms can be used to automatically generate navigation maps. Therefore, robust extraction of geometric features from sensor data is important in the filed of robot navigation.

Hough transform and its efficient variant the LogHough transform are currently the best methods available for extracting line segments from a laser scan. In an indoor

This work was supported by the Australian Research Counci (ARC) and New South Wales Government environment, most of the lines present tend to be aligned with two or three orientations. Therefore searching the Hough space through all possible orientations present is clearly inefficient. In this paper, a parameter invariant to the equation of the line segments is used to segment the range scan and identify potential candidate data points that belong to straight lines. The parameter derived is not sufficiently sensitive to discriminate between straight lines and gradual curves. A Log-Hough transform is therefore used to eliminate false candidates and obtain equations of the lines present. As the search space used for computing the Log-Hough transform is now dramatically reduced, the combined algorithm produces an approximately $30 \%$ reduction in the computational time.

This paper is organized as followed. Section II provides a brief description of existing algorithms for line extraction. The proposed algorithm is developed in section III. Section IV provides practical examples and comparison between the proposed algorithm and traditional Log-Hough techniques. Discussion and conclusions are presented in section $\mathrm{V}$.

\section{EXISTING TECHNIQUES FOR LINE SEGMENT EXTRACTION}

A line can be uniquely described by its slope a, and the intercept with y axis b.

A more robust representation is the use of the perpendicular distance of the line to the origin ( $r$ ) and the angle between a normal to the line and the positive $\mathrm{x}$ axis $(\theta)$, as shown in Figure 1. The equation of the line in $(d, \alpha)$ space is given by

$$
d \alpha=\frac{r}{\cos (\theta-\alpha)}
$$

The simplest method for line extraction is an algorithm adopted from computer vision as described in [2], with variations practically implemented in [3]. The algorithm proceeds iteratively by gradually adding successive laser readings to a line defined by the first few observations. A least square algorithm is used to detect whether the point lies on the postulated line. If the error is greater than the threshold error the line is terminated and another line starts. 


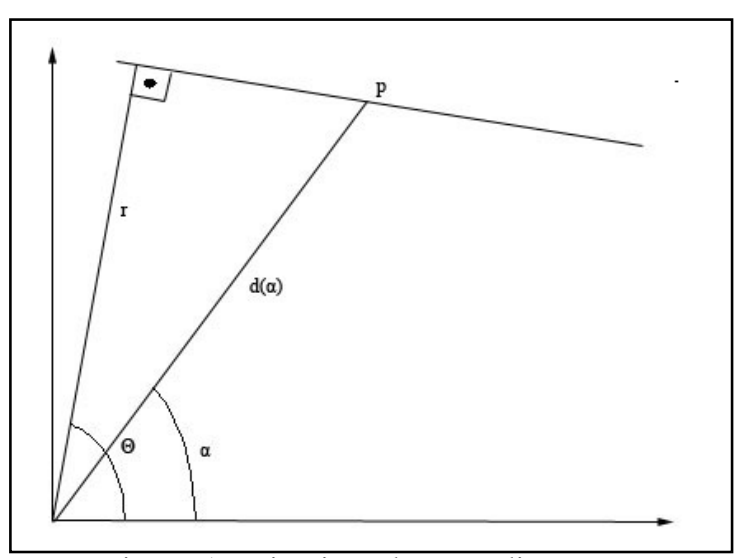

Figure 1 - Line in polar coordinate system

An alternative to the above is iterative endpoint fitting. This method works by joining the first and last point in a laser scan and calculating the orthogonal distance between the points in the scan and the generated line. If this distance is greater then a maximum distance then the line is split into two and the two segments are again analyzed. The algorithm proceeds until the scan is segmented to a set of lines. An alternative implementation of this technique can be found in [3] where initial segmentation into a sequence of data is achieved by examining the distance between successive points and compare this against a threshold. The tolerance is arbitrarily selected as a compromise between the maximum distance at which the depth readings can be taken and the smallest gap between objects the system can detect.

Drawbacks with the above implementations are in selection of a threshold for the error, line length and the inherent recursivness of the algorithms. In addition, if the obtained laser scan is noisy or various objects are obscuring planes defining the map, then the line assembly will fail.

Hough transform technique is significantly more robust then the line fitting strategies discussed above [8]. In the Hough transform, a given observation $(\mathrm{d}, \alpha)$ is mapped to all points in the $\mathrm{r}-\theta$ space that specify a possible line through that point. This set will form a sinusoid. All observations that belong to a particular line will all map to sinusoids that intersect at a common point. The $r-\theta$ space is quantized where $r$ extends over the range $r=\left\{r_{k} \mid r_{k}=r_{0}+\right.$ $\mathrm{k} \Delta \mathrm{r}\}$ and $\theta$ over $\theta=\left\{\theta_{\mathrm{k}} \mid \theta_{\mathrm{k}}=\theta_{0}+\mathrm{k} \Delta \theta\right\}$. An accumulator array $\mathrm{H}(\mathrm{r}, \theta)$ is defined on $\mathrm{r}^{*} \theta$. As each observation $(d, \alpha)$ is mapped to a sampled sinusoid, each accumulator in $\mathrm{H}(\mathrm{r}, \theta)$ along the sinosoid is incremented. When all points are accounted for, the accumulators with the highest count will be taken to indicate the parameters of the line that best explain the points.

The most difficult problem in generating the Hough transform is selecting the quantization levels for $r$ and $\theta$. This not only impacts on the efficiency of the line detector but also the computational requirements [7]. These problems have been addressed in detail in [1], [4] and [5]. Further, the use of Hough transform is restricted to alignment detection; the Hough transform can only determine the polar parameters of the straight lines, not the exact position of the points constituting the line [8].

The quantization problem has been addressed with the Log Hough Implementation where the $r$ space is quantized with a $\log$ distribution utilizing the minimum range $r_{0}$ as the shifting parameter [1]. In addition, performing the operation in $\log$ space also significantly reduces the computational cost of the classic Hough algorithm. LogHough transform is the most effective method currently available for extracting lines. Operation of this algorithm is explained in detail in section III. The major shortcoming of the algorithm is the need to search through the whole range of line orientation while in a typical indoor environment usually the lines are aligned in two principal orientations.

\section{PROPOSED METHOD}

Consider a straight line and three consecutive observations from a scan of a laser manifested as shown in Fig 2.

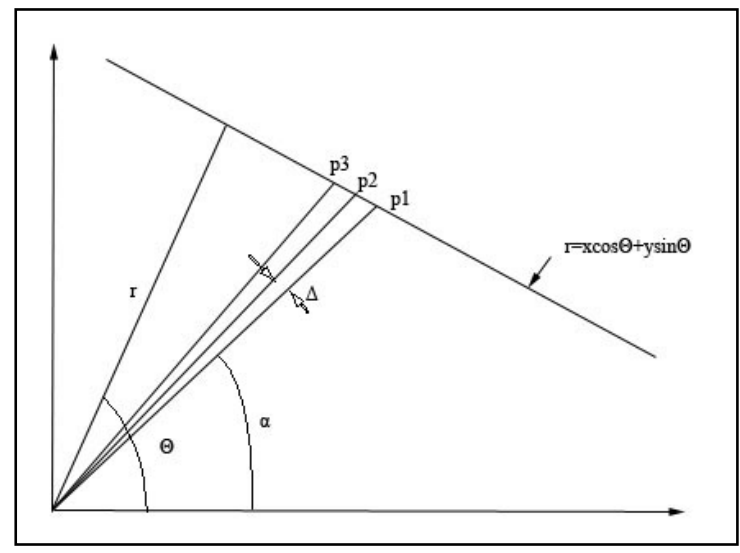

Figure 2 - Laser Scan with Straight Line

The observations are $d_{1}, d_{2}$ and $d_{3}$ where $d_{i}, i=1,2,3$ and $\Delta$ is the angular resolution of the laser rangefinder. From (1)

$d_{i}=\frac{r}{\cos (\theta-\alpha-(i-1) \Delta)}$

Let

$A D=\frac{1 / d_{1}+1 / d_{2}+1 / d_{3}}{1 / d_{2}}$

Substituting (2) in (3) and simplifying gives

$A D=1+2 \cos (\Delta)$ 
It is clear that the parameter $\mathrm{AD}$ is independent of the equation of the straight line and is only a function of the angular resolution of the laser rangefinder. A more generalized form of the parametar AD can be written as

$A D_{i k}=\frac{\sum_{j=-k}^{k} 1 / d_{i-j}}{1 / d_{i}}=1+2 \sum_{j=1}^{k} \cos (j \Delta)$

Clearly $\mathrm{AD}_{\mathrm{ik}}$ for a given observation $\mathrm{p}_{\mathrm{i}}$ is a constant if the points $p_{i-k}$ to $p_{i+k}$ belonging to a straight line. It should be noted here that (4) represent a sufficient condition for $\mathrm{p}_{\mathrm{i}-}$ ${ }_{k}$ to $p_{i+k}$ to belong to a straight line. There are many other curves that give rise to the same value $A D_{i k}$. The most trivial example is an arbitrary curve that passes through all points $\mathrm{p}_{\mathrm{i}-\mathrm{k}}$ to $\mathrm{p}_{\mathrm{i}+\mathrm{k}}$.

Figure 3 shows a scan from a SICK LMS 200 laser rangefinder obtained from an office environment. Figure 4 shows the $\mathrm{AD}_{\mathrm{i} 10}(\mathrm{i}=9, . ., 352)$ for this scan.



Figure 3 - Laser Scan of an office environment

It can be seen from Figure 4 that computing $\mathrm{AD}_{\mathrm{ik}}$ enables identification of potential observations that belong to straight lines within the laser scan. As there is noise always present in the laser scans and that the parameter $A D_{i k}$ is not sufficiently discriminating in the presence of shapes with slight curvature, it is proposed that an efficient variant of the Log-Hough transform is performed to extract the equations of the line segments.

For completeness, the Log-Hough transform is summarized below. For a detailed explanation the reader is referred to [1]. Consider a straight line $r=x \cos \theta+y \sin \theta$ and an inner measurement limit of $r_{o}$

$\log \left[\frac{r}{r_{0}}\right]=\log d-\log r_{0}+\log (\cos (\theta-\alpha))$

where $(\mathrm{d}, \alpha)$ denote single observations from a laser scanner. For all possible values of $\theta, \log \left[r / r_{o}\right]$ can now be computed. Note that if $\log (\cos (\theta))$ is pre-computed and stored then $\log (\cos (\theta-\alpha))$ only involves an indexing operation. Thus the computation involved in (6) is not particularly demanding.

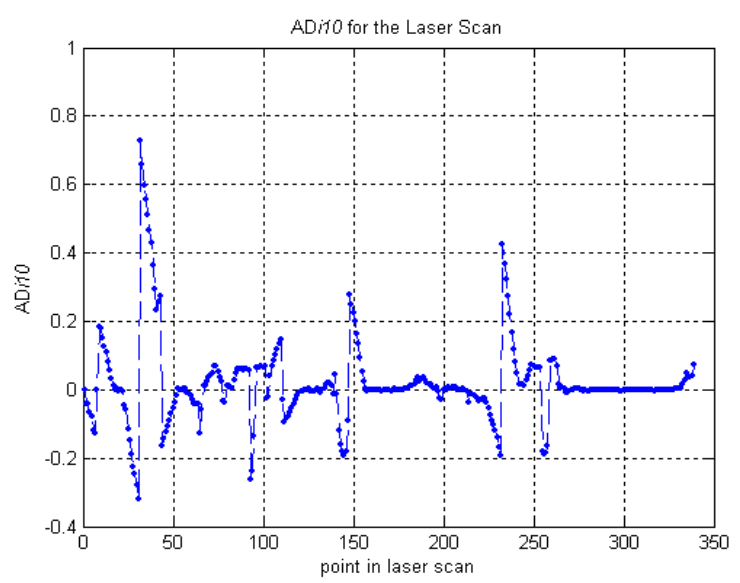

Figure $4-\mathrm{AD}_{\mathrm{i} 10}$ of the Laser Scan shown in Fig 3.

However, in the present example straight line segments are already identified. Therefore, the search space for the Log-Hough transform can be significantly reduced if approximate orientations of the line $(\theta)$ are available.

Consider:

$$
\theta_{i}=\tan ^{-1}\left(\frac{a d_{i k}}{\sum_{j=-k}^{k} \sin (j \Delta)}\right)+\alpha_{i}
$$

Using (2) and simplifying it can be shown that

$$
a d_{i k}=\frac{-\sum_{j=i-k}^{i-1} 1 / d_{j}+\sum_{j=i}^{i+k} 1 / d_{j}}{1 / d_{i}}
$$

Equation (8) can now be used to obtain a value for $\theta$ for the straight line that is defined by points $\mathrm{p}_{\mathrm{i}-\mathrm{k}}$ to $\mathrm{p}_{\mathrm{i}+\mathrm{k} \text {. }}$ Figure 5 illustrates the implementation of (8) on the laser scan data.

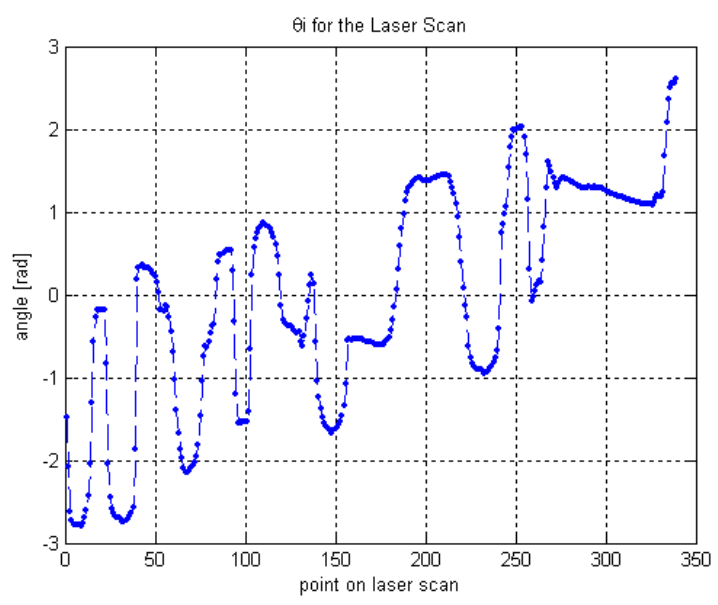

Figure 5 - $\theta$ i for the Laser Scan shown in Fig. 3 
It is now necessary to search only the space surrounding the value for $\theta$ in (8) in the Hough space. It is important to note that all the computations described above are performed in the sensor space $(\mathrm{d}, \alpha)$ without any transformation to the Cartesian co-ordinates $(\mathrm{x}, \mathrm{y})$ thereby resulting in significant reduction in computation time.

To obtain the precise values for $r$ and $\theta$ it is necessary to find the peaks with the highest accumulator count within the Log Hough space Fig 6. Niblack and Petkovic [6] suggest finding the peaks by using a rectangular window that encapsulates the spreading of the peak and produces sub-quantized results of higher accuracy. Once the peak values $r_{\log }$ have been detected these are converted from the quantasized space using (9).

$d=r_{0} e^{r_{\text {log }} * r_{\text {quantize }}}$

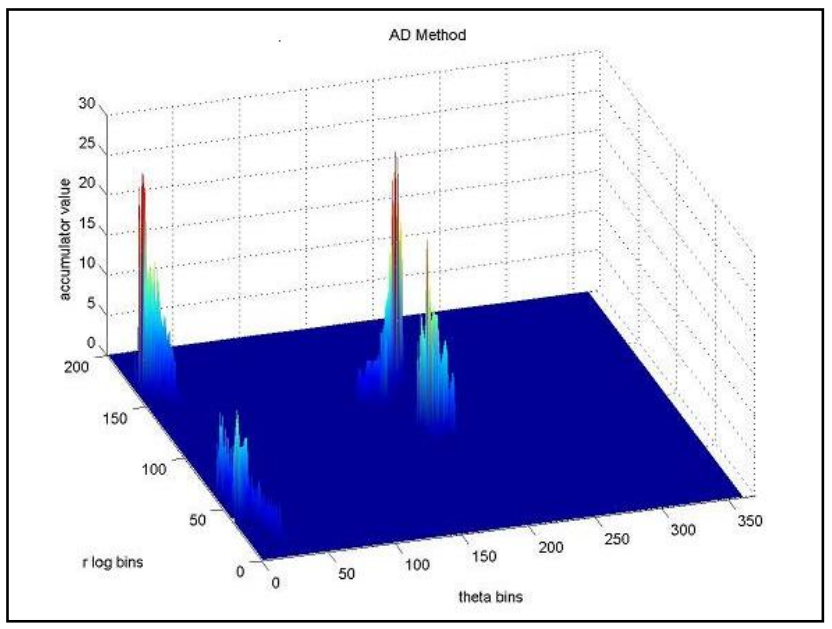

Figure 6 - Proposed Method Log Space

\section{EXPERIMENTAL RESULTS}

The algorithm described above was evaluated using two sets of experimental data. The first data set was obtained from a Pioneer Robot equipped with a SICK LMS 200 laser rangefinder maneuvering in the UTS Laboratory. The second data set has been obtained from the Robotics Data Set Repository - Radish [9] and contains data from s test conducted at the Intel Laboratory - Seattle, seen in Fig 7.

In both data sets the laser rangefinder can produce the bearing and range scans at 0.5 degrees separation. $\mathrm{k}=10$ was used as the smoothing parameter. Hence from (5) $\mathrm{AD}_{\mathrm{ik}}$ $=1+9.9892$. A threshold of \pm 0.015 around this value was empirically selected to determine the points that constitute a line. This threshold was been empirically selected. All points that have $\mathrm{AD}_{\mathrm{ik}}$ less than the threshold and surrounding \pm 5 points were selected. To obtain the estimated angle $\theta$, all individual $\theta$ 's obtained for every point $\mathrm{p}_{\mathrm{i}-10}$ to $\mathrm{p}_{\mathrm{i}+10}$ on the line.

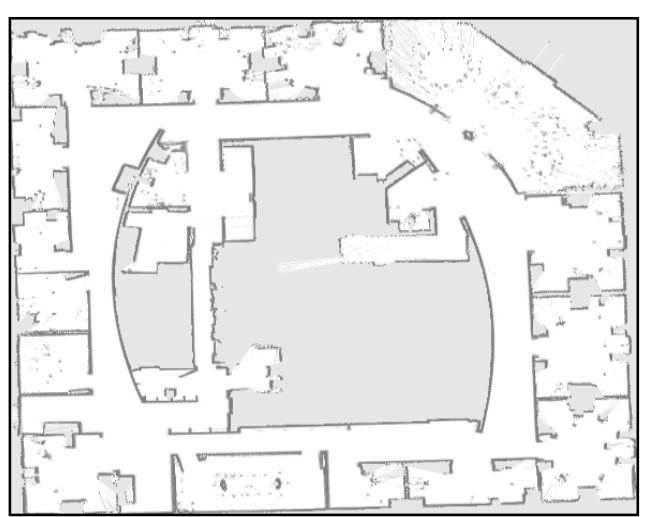

Figure 7 - Map Intel Laboratory Seattle

The comparison between the line segments detected using the proposed algorithm and the traditional Log Hough transform is illustrated in Fig 8 for the UTS data set and Fig 9 for the Intel Laboratory Set. As expected, both algorithms produce similar results. Table 1 contains an comparison between the detected line attributes $\mathrm{r}$ and $\theta$ using the alternative methods for the Intel Laboratory Set illustrated in Fig 9.

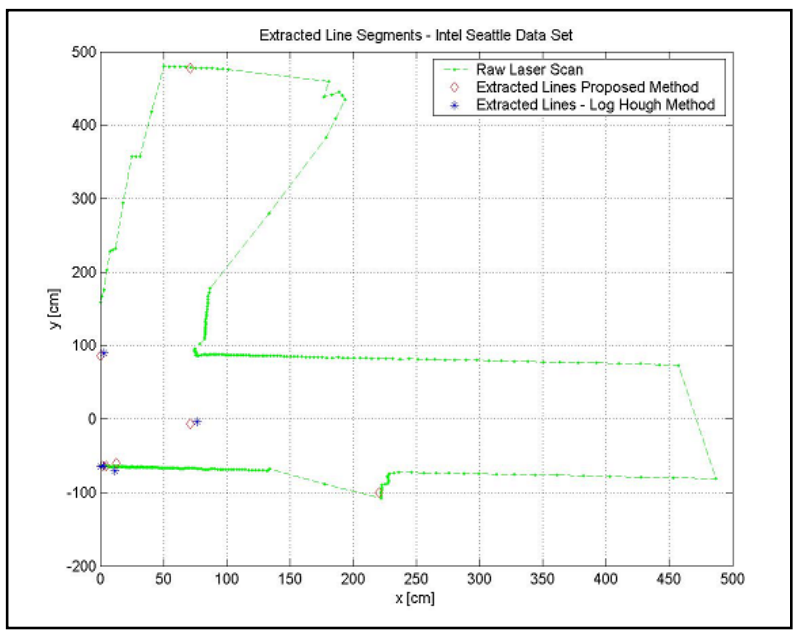

Figure 8 - Extracted Line Segments, UTS Laboratory Data Set

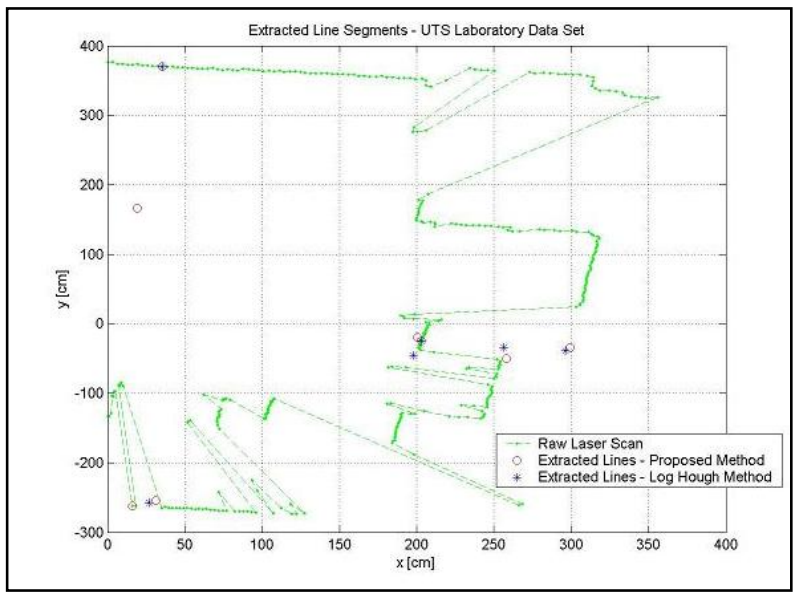

Figure 9 - Extracted Line Segments, Intel Laboratory Seattle Data Set 


\begin{tabular}{|c|c|c|c|}
\hline \multicolumn{4}{|c|}{ Detected Line Segments } \\
\hline \multicolumn{2}{|c|}{ Log Hough Method } & \multicolumn{2}{|c|}{ Proposed Method } \\
\hline $\boldsymbol{r}[\mathbf{c m}]$ & $\boldsymbol{\theta}[\mathrm{rad}]$ & $\boldsymbol{r}[\mathrm{cm}]$ & $\boldsymbol{\theta}[\mathrm{rad}]$ \\
\hline-1.57 & 89.9 & -1.57 & 85.9 \\
\hline & & -0.43 & 242.1 \\
\hline-0.05 & 76.6 & -0.08 & 71.4 \\
\hline-1.53 & 65.2 & -1.5 & 63.7 \\
\hline-1.4 & 71.4 & -1.36 & 71.5 \\
\hline & & 1.42 & 483.1 \\
\hline-1.54 & 64.4 & -1.54 & 64.4 \\
\hline
\end{tabular}

Table 1 - Comparison between Log Hough and Proposed Method

A comparison between the computational time required for extraction of line segments in Fig. 10 demonstrates the efficiency of the proposed method in comparison to the Log Hough implementation for the identical data set.

The variation of the computational time seen for the Log Hough method is due to the fact that all scans do not have identical number of observations as the laser scan has a limited range. Proposed algorithm has a computational time that is a function of the number of straight line segments present in the environment

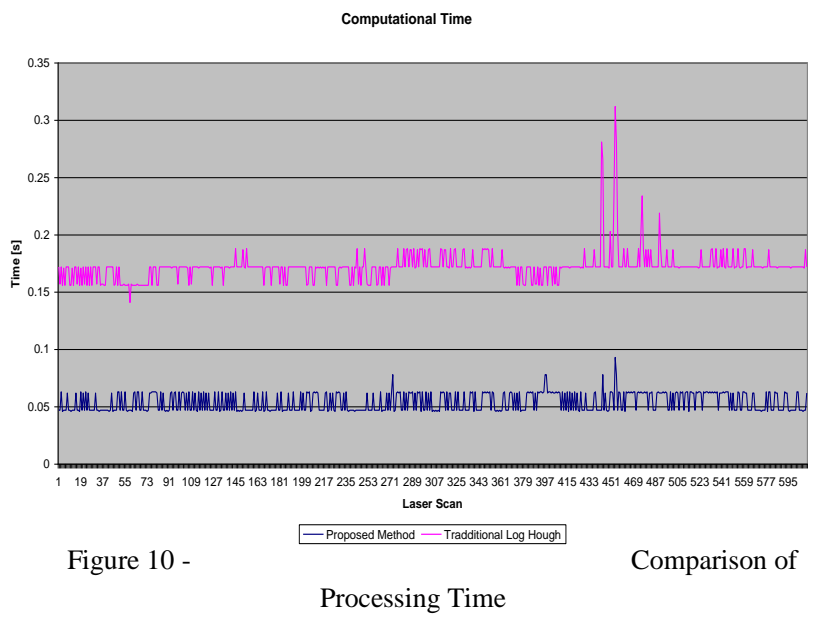

\section{CONCLUSIONS}

The main contribution of this paper is a new method for extracting line segments from a laser scan. The approach is based on using a pose-invariant parameter, computed in the sensor space, to segment a laser scan and identify likely candidates that may belong to straight lines. The segmented scan is then processed using a Log Hough transform to obtain the equations of the lines. Another parameter that is also computed in the sensor space is derived for obtaining the approximate orientation of the lines present, thereby significantly reducing the size of the window the needs to be searched during the Hough transform. This parameter may be further used to identify the exact position of the points constituting the line. Thus, the proposed method enables robust and efficient line extraction from laser range data.

\section{ACKNOWLEDGMENT}

This work is supported by the ARC of Excellence programme, funded by the Australian Research Council (ARC) and the New South Wales Government.

\section{REFERENCES}

[1] Giesler, B.; Graf, R.; Dillmann, R.; Weiman , "Fast mapping using the Log-Hough transformation", Intelligent Robots and Systems, 1998. Proceedings., 1998 IEEE/RSJ International Conference on ,Volume: 3, 13-17 Oct. 1998 , Pages:1702 - 1707 vol.3

[2] P.E Hart, R.O Duda. "Pattern Classification and Scene Analysis" John Wiley and Sons, 1973

[3] J. Crowley, "Navigation for an intelligent mobile robot"; IEEE Journal of Robotics and Automation, Volume: 1, Issue: 1, Mar 1985

[4] D. Leung, L. Lam, and W. Lam, "Diagonal Quantization of the Hough Transform", Pattern Recognition Letters, vol. 14, pp. 181189,1993

[5] W. Lam, L. Lam, K. Yuen, and D. Leung, aAn Analysis on Quantizing the Hough Space”, Pattern Recognition Letters, vol. 15, pp. 1,127-1,135, 1994.

[6] Niblack, W.; Petkovic, D, "On improving the accuracy of the Hough transform: theory, simulations, and experiments"; Computer Vision and Pattern Recognition, 1988. Proceedings CVPR '88., Computer Society Conference on , 5-9 June 1988, Pages:574 - 579

[7] Svalbe, I.D.; "Natural representations for straight lines and the Hough transform on discrete arrays" IEEE Transactions on Pattern Analysis and Machine Intelligence ,Volume: 11 , Issue: 9 , Sept. 1989 Pages:941 - 950

[8] Kesidis, A.L.; Papamarkos, N. "On the inverse Hough transform"; Pattern Analysis and Machine Intelligence, IEEE Transactions on, Volume: 21 , Issue: 12 , Dec. 1999 Pages:1329 - 1343

[9] Radish: The Robotics Data Set Repository, Standard data sets for the robotics community. http://radish.sourceforge.net , last viewed July $10,2004$. 